\title{
Parental stress around ophthalmological health conditions: a systematic review of literature protocol
}

\author{
Julio Cesar Souza-Silva ${ }^{*}$ (D) Cleusa Alves Martins², Marco Túlio Antônio Garciazapata ${ }^{3}$ and Maria Alves Barbosa ${ }^{2}$
}

\begin{abstract}
Background: Parents can be psychologically impacted when their children are diagnosed with eye diseases, such as blindness, strabismus, and eye cancer. Stress can reduce the quality of parental care and may be linked to the deterioration of parents' and children's mental and physical health and family dynamics. No systematic literature review on parental stress in ophthalmology has been found to provide evidence synthesis capable of stimulating and defining new studies and thereby promoting research in this field. To address this important gap, the present review aims to synthesize evidence about approaches, methods, instruments, and results from research regarding ophthalmologyrelated parental stress.
\end{abstract}

Methods: Primary epidemiological observational studies should be original in addressing parental stress caused by ophthalmological health conditions in children. They should present the characteristics of the study population and the clinical and ophthalmic characterizations of children.

MEDLINE (via Ovid), EMBASE, PsycINFO, Google Scholar, and gray literature (PsycEXTRA, NTIS, and OpenSINGLE) will be searched. Controlled vocabulary, Boolean operators, and defined search strategies will be used. There will be no restrictions on the studies' publication language, which will be selected in two screening stages. Two reviewers will independently retrieve full-text studies, assess methodological quality, and extract data. Data available through December 2021 will be considered for inclusion.

Discussion: The socioeconomic characterization of the participants, the identification of which ophthalmological diseases have been studied in relation to parental stress, and the knowledge of each instrument and methodology peculiarities potentially contribute to this study. The results may promote the development or enhancement of public policies focused on this specific theme, thereby providing the means for potential improvement of the physical and mental health of parents and children with eye diseases.

Systematic review registration

PROSPERO CRD42018094972

Keywords: Parenting Stress, Ophthalmology, Ocular Diseases, Systematic Review, Eye Diseases

\footnotetext{
${ }^{*}$ Correspondence: julio.cesar@ufg.br

${ }^{1}$ Faculty of Medicine, Post-Graduate Program in Health Science, Federal University of Goiás, Secretaria-1a s/n-Setor Universitário, Goiânia, Goiás 74605-020, Brazil

Full list of author information is available at the end of the article
}

\section{Background \\ Description of the disease}

The World Health Organization estimates that 1.4 million children-defined as individuals between 0 and 15 years of age-are blind, with a prevalence of $0.3 / 1000$ children in developed countries and 1.5/1000 in poor/very poor

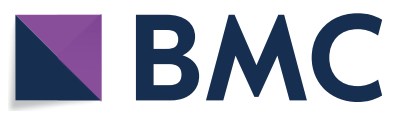

(c) The Author(s) 2021. Open Access This article is licensed under a Creative Commons Attribution 4.0 International License, which permits use, sharing, adaptation, distribution and reproduction in any medium or format, as long as you give appropriate credit to the original author(s) and the source, provide a link to the Creative Commons licence, and indicate if changes were made. The images or other third party material in this article are included in the article's Creative Commons licence, unless indicated otherwise in a credit line to the material. If material is not included in the article's Creative Commons licence and your intended use is not permitted by statutory regulation or exceeds the permitted use, you will need to obtain permission directly from the copyright holder. To view a copy of this licence, visit http://creativecommons.org/licenses/by/4.0/. The Creative Commons Public Domain Dedication waiver (http://creativeco mmons.org/publicdomain/zero/1.0/) applies to the data made available in this article, unless otherwise stated in a credit line to the data. 
communities. These children face a lifetime of blindness, which equates to a combined estimated 75 million blind years (blind individuals $\times$ life expectancy) [1] .

Each year, 500,000 children (approximately one per minute) are born blind or become blind before their fifth birthday. The causes of blindness, which vary according to geographic region and socioeconomic status, include corneal scarring, cataracts, glaucoma, retinopathy of prematurity, and refractive errors. Although eye disease may not always lead to low vision or blindness, it can still cause significant parental stress stemming from the processes of diagnosis, treatment, and rehabilitation of vision. In all such cases, parental involvement and understanding are of paramount importance [1].

Being the parent of a child with any chronic disease can cause stress $[2,3]$, which is defined by Hans Selye as "a non-specific body response to any demand made upon it" [4]. Parental stress refers to a series of processes that lead to psychological and physiological reactions when attempting to adapt to parenting activities [3]. Early identification of increased stress in the parent-child system and suitable interventions can help reduce stress and diminish the frequency and intensity of the child's emotional and behavioral disorders [5]. The reduction of parental stress improves parental health [6] and positively impacts the quality of care provided to sick children [7].

Conversely, increasing and chronic levels of parental stress may put parents, children, and other family members at risk of adverse physical and psychological effects, such as anxiety and depression [8]. Among children, prolonged stress can lead to social incompetence, maladaptive behaviors, and cognitive impairment [2]. Stressful events often influence the pathogenesis of physical illness, causing negative affective states. In turn, these directly impact biological processes or care patterns, increasing the risk of becoming sick [9].

Changes in care may occur due to caregivers' adaptations or coping responses to stress, such as increased smoking, decreased physical activity, insomnia, disinterest, and poor adherence to medical prescriptions and healthy habits. Furthermore, stress has been linked to major depression and poor adherence to treatments [9].

There are records of interference in the parent-child system due to parental stress around a child's ophthalmological issues, but we did not find any systemic literature reviews on the subject. The current published studies on psychological/psychiatric diseases in the ophthalmological field [10-17] mainly focus on depression, anxiety, and parental burden; they do not address parental stress and child with visual disorders. A systematic literature review aimed at generating evidence and synthesis of what has been studied and published is required to support the conception and planning of new studies. The evidence synthesis will guide new research and promote further studies to improve care for children with eye diseases along with the physical and mental health of their parents.

\section{Eye disorders in children and psychological implications for parents and family}

During pregnancy, parents idealize the birth of a perfect child [18]. The birth of a blind child with strabismus, glaucoma, or congenital eye diseases can create a discrepancy between the idealized and the real child. One aspect of motherhood necessary to establish a healthy mother-child relationship is being able to deal with such discrepancies. Failure in overcoming expectation-reality discrepancies can lead mothers to become depressed, distance themselves from the child, and become unable to provide the warmth and love required to promote the child's healthy development [10].

The establishment of an official diagnosis of a child with disability (e.g., blindness) marks the occurrence of a family crisis, where members start expressing feelings of sadness, anger, guilt, helplessness, and isolation. Stress can be linked to the disruption of what was idealized versus the reality and the breakdown of family routine, and parents with very high levels of stress are required to be guided to enable them to effectively provide higher levels of care [19].

The possibility of parental stress becoming an agent of change in the relationship between a parent and a child raises questions as to what levels of resilience and coping strategies must be adopted by children's families to alleviate the possible psychological distress caused by children's eye diseases. It is unclear whether stress associated with the basal parental role of daily life is sufficiently robust to lead to clinical disturbances or whether the parental stress experienced by the parents of children with eye diseases correlates with psychological conditions that are potentially harmful to the parents, child, or family health.

More research about parental stress related to ophthalmological disorders in children is necessary because (1) blindness affects over a million children worldwide, (2) stress is a known contributor to many serious health issues, and (3) quality of life and appropriate allocation of healthcare resources are high-priority issues. Systematic reviews will help translate knowledge into action and promote more relevant studies. The first step to progress research in this field is to create a protocol for the synthesized evidence on the methodology used by researchers to evaluate parental stress related to children's eye diseases, the research instruments, the psychometric characteristics of these conditions, and study 
design advantages and disadvantages, limitations, and peculiarities.

\section{Review questions}

The review questions for this systematic review of literature are as follows:

i. What approaches have been used to research parental stress resulting from pediatric eye disease?

ii. What methodologies are employed in studies of parental stress resulting from pediatric eye diseases?

iii. What parental stress assessment tools are used in pediatric eye disease research?

iv. What peculiarities and psychometric characteristics of parental stress assessment instruments are employed in pediatric eye disease research?

v. What are the main results of research conducted on parental stress resulting from pediatric eye diseases?

\section{Objectives}

To identify studies and their respective authors as well as the bibliographical references related to parental stress in ophthalmology

To characterize the sociodemographic aspects of participants in studies on parental stress in ophthalmology as well as the clinical and ophthalmic conditions of their children

To identify the methodological design and timeline of selected studies on parental stress in ophthalmology

To describe the methodology of the instrumentif present-used to measure parental stress, with emphasis on its psychometric characteristics (internal reliability, test and retest reliability, and validation criteria)

To highlight the main results and conclusions of authors of published research on parental stress around ophthalmology

To detect the peculiarities of each study presented in the research on parental stress around ophthalmology

\section{Inclusion criteria}

Population, exposure, comparator, outcome, and study design components (PECOS) to be analyzed in the studies are as follows:

i. Population/participants: father, mother, or both ii. Exposure of interest: eye disease in the child resulting from severe reduction in visual acuity, dysfunction of extrinsic eye motility, cancer, congenital eye diseases, deforming/stigmatizing eye, and periocular changes or any eye disease with the potential to increase parental stress

iii. Comparator: no specific comparator

iv. Outcome: parental stress

v. Study design: primary epidemiological observational studies

Studies should be original in addressing parental stress (only father, mother, or both parents) caused by eye disease in children. The terms "parental stress" or "parenting stress" or similar terms describing this condition must be included in the title, summary, or the full text. Characterization of the study population and clinical and ophthalmic characterization of the children should be present. Regarding the type of research, laboratory experimental studies, reviews, editorials, comments, mathematical models, methodological articles, expert opinions, and other methodological modalities will be excluded due to the methodological differences that may hinder comparison across studies. Studies on parental stress in caregivers or other family members will also not be eligible. Similar studies that do not meet all the inclusion criteria, but that have relevant information, will be analyzed separately in the discussion section of the systematic review.

\section{Methods}

The proposed literature review will be conducted in accordance with the Joanna Briggs Institute methodology for systematic reviews of observational epidemiological studies that report prevalence and cumulative incidence data [20].

\section{Study record}

This systematic literature review protocol is an integral part of a doctoral dissertation, entitled "Parental Stress in Mothers and Quality of Life of Infants and Blind Children." It has been registered in the international database PROSPERO (under registration number CRD42018094972) and is being reported according to the Preferred Reporting Items for Systematic Reviews and Meta-Analyses Protocols (PRISMA-P) [21, 22] (see checklist in Additional File 1).

\section{Information sources}

The research strategy will be developed using Medical Subject Headings (MeSH) and Elsevier Life Science Thesaurus (Emtree) descriptors. Reviewers (JCSS and CAM) will systematically search the following databases: 
MEDLINE (via Ovid), EMBASE, PsycINFO, and Google Scholar.

Other data sources, such as ProQuest Dissertations and Theses, databases, and gray literature (i.e., PsycEXTRA, NTIS and OpenSINGLE), will also be consulted. Data will be considered for inclusion until December 2021.

\section{Search strategy}

Searches will use a controlled vocabulary defined by initial search terms. Examples of search terms and search strategies are presented in Additional File 2. The snowballing method [23] will be used to identify other studies from the references of selected articles. Search terms in Emtree will be matched with equivalent terms in MEDLINE, to be able to conduct a search in EMBASE. Searches will be designed to be conducted in MEDLINE and EMBASE and will be adapted to other electronic databases and gray literature, always aiming to achieve search equivalence. There is no time limit filter for the inclusion of articles in all databases. The search strategy will be developed by an information science expert, aiming at conducting a high specificity and sensitivity search for the review.

\section{Study selection}

Studies should be original in addressing parental stress (only father, mother, or both parents) caused by eye disease in children. Parental stress (or parenting stress) must be written in the title, summary, or the full text. Additionally, the characterization of the study population and clinical and ophthalmic characterization of the children should be present. The study search will be conducted by two independent researchers [24] in a two-stage selection process.

There will be no blind strategy for reviewers regarding authors' names, institutional affiliations, or country of origin of the analyzed studies.

The raw data obtained from the databases will be processed and duplicate studies eliminated using the ENDNOTE $^{\circledR}$ (ENDNOTE X7, Thomson Reuters, USA) reference manager program.

\section{Selection phase I}

In the first phase, the articles will undergo Relevance Test 1 (RT-1); reviewers will analyze the title and abstract of the article, determining whether it fulfills the eligibility criteria listed in Table 1.

\section{Selection phase II}

After meeting the RT- 1 criteria, studies will be subjected to Relevance Test 2 (RT-2). This verifies, more strictly, if there is presence of full text, characterization of the study population, and clinical and ophthalmic characterization of the children (see RT-2 in Table 2).

Excluded studies will be recorded in a separate table along with the reason for exclusion. "Results" will include a comment section containing the characteristics of each excluded study and the reason that led to their exclusion.

In phases I and II, articles having items evaluated as "not clear" will be the objects of a joint analysis among reviewers. In cases where disagreement persists, a third reviewer will provide his/her opinion essential

Table 1 Relevance Test 1 (RT-1) of studies included in the systematic review

\begin{tabular}{l}
\hline \\
\hline Is the research original? \\
Does the population include only fathers, only mothers, or both parents? \\
Does the child have an eye disease/condition? \\
Does the child's condition result in parental stress? \\
Is the child between 0 and 12 years old? \\
Does the child have another chronic/disabling condition?
\end{tabular}

Table 2 Relevance test 2 (RT-2) for the systematic literature review

\begin{tabular}{l}
\hline \\
\hline Is there an instrument that specifically assesses parental stress? \\
Is the full text of the article available in databases? \\
Is there a characterization of the study population? \\
Is there a methodological characterization of the instrument? \\
Is there a clinical and ophthalmic characterization of the children? \\
\hline
\end{tabular}


to the resolution of the case. Studies that meet all the inclusion criteria will be accepted for research. The agreement achieved among the reviewers of this systematic review will be assessed using Cohen's $(k)$ kappa agreement index [25].

The results of the search will be reported in full in the final report and presented in a Preferred Reporting Items for Systematic Reviews and Meta-analyses (PRISMA) flow diagram (see Fig. 1) [26].

\section{Assessment of methodological quality}

The quality of the included articles will be assessed using the Joanna Briggs Institute assessment tool for methodological guidance for systematic reviews of observational epidemiological studies reporting prevalence and cumulative incidence data (see Additional File 3) [20].

The methodological quality of the included articles will be assessed by two reviewers. In case of disagreement regarding the quality of a particular study, a third reviewer will be the tiebreaker, providing the final assessment. There is no intention of conducting a meta-analysis of the data.

\section{Data extraction and management Data extraction}

Data will be extracted from papers included in the systematic review by the two independent reviewers using a data extraction tool developed by the reviewers. In this phase, the text of the article will be analyzed in full, elaborating a standardized cataloging form to record product information (see form in Additional File 4).

Additional relevant information not foreseen in the fields above and the peculiarities of each study will be recorded in the "observations" field.

If some information or detail is missing from the article, the study will be included, although the absence of data will be noted.

In case of lack/loss/uncertainty of information, the author of the study will be contacted by email three times. If the problem persists, the study will be excluded.

\section{Data synthesis}

Since our study focuses on methodological analysis by assessing approaches, methodologies, instrument characteristics, and psychometric characteristics, there will be no combination of individual outcomes-that is, no statistical calculations or meta-analysis of results will be performed. Heterogeneity will be verified through the analysis of subgroups and configurations (country,

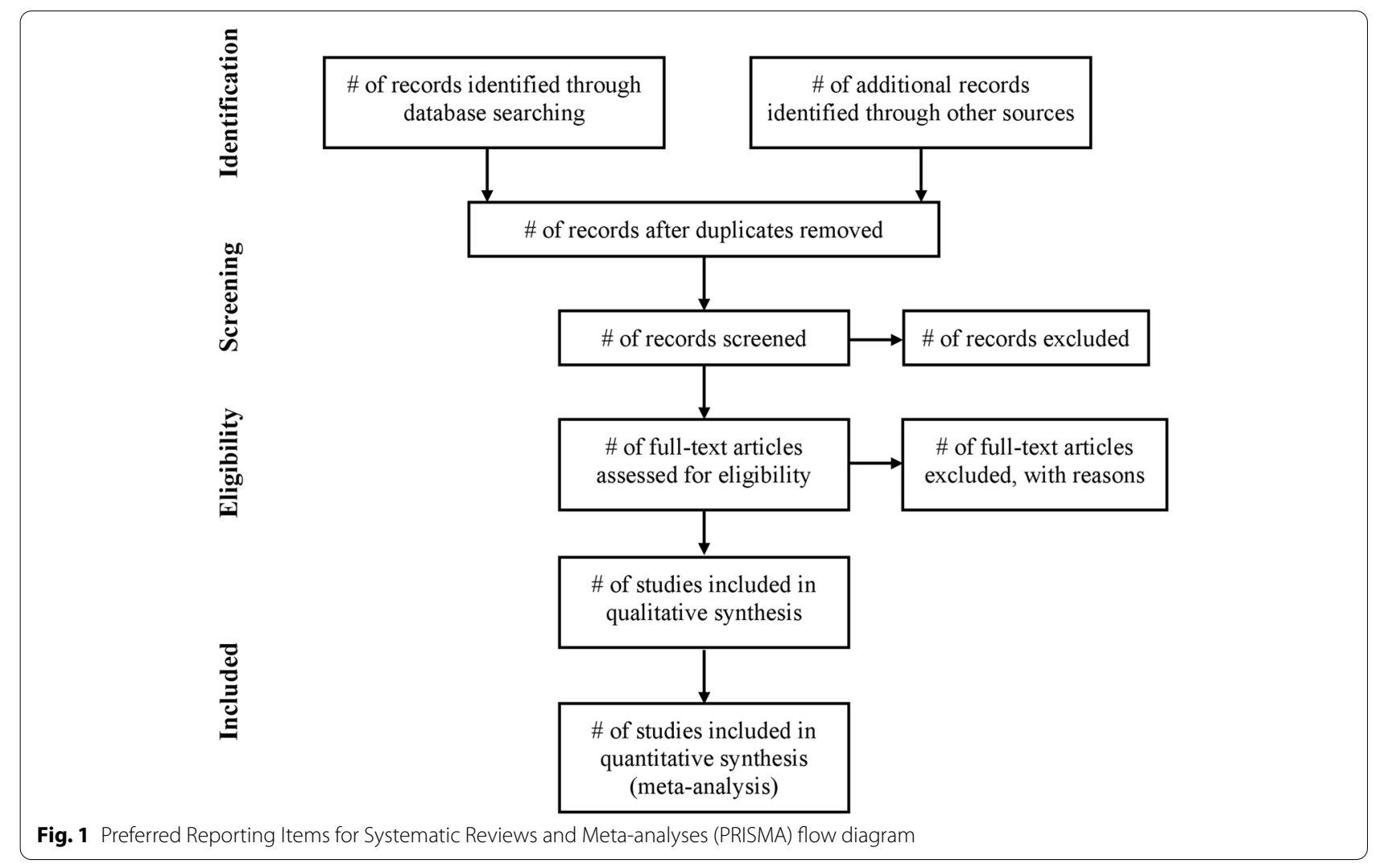


hospital, clinic, etc.), conditions (complete blindness, sudden onset versus gradual loss of vision), population (children, preschool age, adolescents), observing possible changes in the approach or in the type of instrument to be administered to measure stress. This will generate a measurement capable of summarizing the outcomes. We will critically analyze the data and quality of the studies included, providing descriptive tabulations and summaries. The results extracted from the cataloging form will be categorized and compared between the different studies. If any included studies show important methodological differences, a sensitivity analysis will be carried out. Gaps and limitations in the description or methodology will be noted and discussed.

\section{Protocol changes}

Any substantive change to this protocol will be recorded upon its occurrence, in PROSPERO, and documented in the final publication.

\section{Discussion}

To our knowledge, this will be the first systematic literature review related to the comparative assessment of approaches, methods, and results in the research on parental stress around ophthalmology. Systematic analysis and synthesis of peculiarities, time of administration, cost, reliability, and stability can offer researchers support for instrument analysis for choosing the best match for their research. Psycho-ophthalmology is a field linking ophthalmology and psychiatry [27]. Few studies related to parental stress have been conducted on this subject. The socioeconomic characterization of the participants, the identification of which ophthalmological diseases have been studied in relation to parental stress, and the knowledge of each instrument and methodology peculiarities potentially contribute to this study. The results may promote the development or enhancement of public policies focused on this specific theme, thereby providing the means for the potential improvement of the physical and mental health of parents of and children with eye diseases.

This review protocol may have potential limitations if attempted to predict the evaluation of psychometric characteristics, such as equivalence and construct analysis [28]; however, as it does not attempt to do so, it keeps the specific evaluation as the object of study of future research.

\footnotetext{
Abbreviations

CAPES: Coordenação de Aperfeiçoamento de Pessoal de Nível Superior (Coordination for the Improvement of Higher Education Personnel); FAPEG: Fundação de Amparo à Pesquisa do Estado de Goiás (Research Support Foundation of the State of Goiás).
}

\section{Supplementary Information}

The online version contains supplementary material available at https://doi. org/10.1186/s13643-021-01773-8.

Additional file 1. PRISMA-P Checklist. This file contains the completed PRISMA-P checklist.

Additional file 2. Search strategy and search terms for MEDLINE (Ovid). This file presents the search strategy and terms to be used in the review.

Additional file 3. Joanna Briggs Institute assessment tool for methodological guidance for systematic reviews of observational epidemiological studies reporting prevalence and cumulative incidence data. This file presents the Joanna Briggs Assessment of Methodological Quality Tool.

Additional file 4. Standardized form for data collection. This file presents the form for data collection.

\section{Acknowledgements}

This study was financed by the Coordination for the Improvement of Higher Education Personnel (CAPES) and the Research Support Foundation of the

State of Goiás (FAPEG), providing a Ph.D. scholarship to the first author (reference number 88887.295574/2018-00).

This study received financial support for publication from the Federal University of Goiás (UFG).

We are grateful to Pollyanna Moreira, who acted as the research assistant and was responsible for collecting data from the mothers.

\section{Authors' contributions}

JCSS is the review guarantor and first reviewer, working on all phases of the systematic literature review. CAM is the second reviewer. MAB and CAM contributed to the elaboration of the protocol, research question, research strategy, and final revision of the protocol text. MTAG contributed to the process of evaluation and synthesis of evidence quality. MAB and MTAG will compose the arbitration panel and complete the revision of the protocol text. The authors read and approved the final manuscript.

\section{Authors' information}

Not applicable.

\section{Funding}

This systematic literature review was funded by the authors themselves.

Availability of data and materials

Not applicable.

\section{Declarations}

Ethics approval and consent to participate

This systematic review is an integral part of a doctoral dissertation and the research project was approved by the Research Ethics Committee of Clinical Hospital of the Federal University of Goiás and published in Plataforma Brasil CAAE 90833718.2.0000.5078 under opinion number 2.746.572.

Consent for publication

Not applicable.

Competing interests

The authors declare that they have no competing interests.

\section{Author details}

${ }^{1}$ Faculty of Medicine, Post-Graduate Program in Health Science, Federal University of Goiás, Secretaria-1a s/n-Setor Universitário, Goiânia, Goiás 74605-020, Brazil. ' 'Faculty of Nursing, Federal University of Goiás, Rua 227 gd. 68 Setor Leste Universitário, Setor Leste Universitário 74605080, Goiânia, GO, Brazil. ${ }^{3}$ Department of Tropical Medicine and Dermatology, Institute of Tropical Pathology and Public Health, Rua 235/Esq. com 1a Avenida, Setor Universitário 74605050, Caixa-postal: 131, Goiânia, GO, Brazil. 
Received: 14 January 2020 Accepted: 26 July 2021

Published online: 13 August 2021

\section{References}

1. World Health Organization. Global Initiative for the Elimination of Avoidable Blindness: action plan 2006-2011. Geneva: World Health Organization; 2007.

2. Golfenshtein N, Srulovici E, Medoff-Cooper B. Investigating Parenting Stress Across Pediatric Health Conditions - A Systematic Review, Comprehensive Child and Adolescent Nursing. 2016;39(1):41-79. https://doi.org/ 10.3109/01460862.2015.1078423.

3. Deater-Deckard K, Panneton R. Parental stress and early child development: adaptive and maladaptive outcomes. Cham: Springer; 2017.

4. Selye H. The evolution of the stress concept. Am Sci. 1973;61:692-9.

5. Abidin RR. Parenting Stress Index ${ }^{\mathrm{TM}},\left(\mathrm{PSI}^{\mathrm{TM}}-4\right)$ professional manual. 4 ed. Lutz, Florida: Par Inc:; 2012.

6. Golfenshtein N, Srulovici E, Deatrick JA. Interventions for reducing parenting stress in families with pediatric conditions: an integrative review. J Fam Nurs. 2016;22:460-92.

7. Chaplin TM, Turpyn CC, Fischer S, et al. Parenting-Focused Mindfulness Intervention Reduces Stress and Improves Parenting in Highly Stressed Mothers of Adolescents. Mindfulness. 2021;12:450-62. https://doi.org/10. 1007/s12671-018-1026-9.

8. Vismara L, Rolle L, Agostini F, Sechi C, Fenaroli V, Molgora S, et al. Perinatal parenting stress, anxiety, and depression outcomes in first-time mothers and fathers: a 3- to 6-months postpartum follow-up study. Front Psychol. 2016:7:938.

9. Cohen S, Janicki-Deverts D, Miller GE. Psychological stress and disease. JAMA. 2007:298(14):1685-7.

10. Tolchin JG, Lederman ME. Congenital (infantile) esotropia: psychiatric aspects. J Pediat Ophth Strab. 1978;15(3):160-3.

11. Bambara JK, Owsley C, Wadley V, Martin R, Porter C, Dreer LE. Family caregiver social problem-solving abilities and adjustment to caring for a relative with vision loss. Invest Ophthalmol Vis Sci. 2009;50(4):1585-92.

12. Duman NS, Gökten ES, Duman R, Duman R, Çevik SG. Evaluation of depression and anxiety levels in mothers of babies' following due to premature Rrtinopathy. Arch Psychiat Nurs. 2018;32(3):439-43.

13. Hahm B-J, Shin Y-W, Shim E-J, Jeon HJ, Seo J-M, Chung H, et al. Depression and vision-related quality of life in patients with retinitis pigmentosa. Brit J Ophthalmol. 2008;92(5):650-4.

14. Zhang X, Bullard KM, Cotch MF, Wilson MR, Rovner BW, McGwin G Jr, et al. Association between depression and functional vision loss in persons 20 years of age or older in the United States, NHANES 2005-2008. JAMA Ophthalmol. 2013;131:573-81.
15. Drews C, Celano M, Plager DA, Lambert SR. Parenting stress among caregivers of children with congenital cataracts. J Am Assoc Pediat Ophthalmol Strab. 2003;7(4):244-50.

16. Celano M, Hartmann EE, Drews-Botsch CD. Parenting stress in the infant aphakia treatment study. J Pediatr Psychol. 2013;38(5):484-93.

17. Wang $L$, Zhong WX, Ji XD, Chen J. Depression, caregiver burden and social support among caregivers of retinoblastoma patients in China. Int J Nurs Pract. 2016;22:478-85.

18. Solnit AJ, Stark MH. Mourning and the birth of a defective child. Psychoanal Study Child. 1961;16:523-37.

19. Fortier $L M$, Wanlass RL. Family crisis following the diagnosis of a handicapped child. Fam Relat. 1984:13-24.

20. Munn Z, Moola S, Lisy K, Riitano D, Tufanaru C. Methodological guidance for systematic reviews of observational epidemiological studies reporting prevalence and cumulative incidence data. Int J Evid Based Healthc. 2015;13:147-53.

21. Moher D, Shamseer L, Clarke M, Ghersi D, Liberati A, Petticrew M, et al. Preferred reporting items for systematic review and meta-analysis protocols (PRISMA-P) 2015 statement. Syst Rev. 2015;1(4):1.

22. Shamseer $L$, Moher $D$, Clarke $M$, Ghersi D, Liberati A, Petticrew $M$, et al. Preferred reporting items for systematic review and meta-analysis protocols (PRISMA-P) 2015: elaboration and explanation. BMJ (Clinical research ed). 2015;350:g7647.

23. Tsafnat G, Glasziou P, Choong MK, Dunn A, Galgani F, Coiera E. Systematic review automation technologies Syst Rev. 2014;3:74.

24. Higgins J. Green S. Cochrane handbook for systematic reviews of interventions version 5.1. 0. The Cochrane Collaboration. Confidence intervals. 2011.

25. Cohen J. Weighted kappa: nominal scale agreement provision for scaled disagreement or partial credit. Psychol Bull. 1968;70:213-20.

26. Liberati A, Altman DG, Tetzlaff J, Mulrow C, Gøtzsche PC, loannidis JP, et al. The PRISMA statement for reporting systematic reviews and meta-analyses of studies that evaluate health care interventions: explanation and elaboration. PLoS Med. 2009;6(7):e1000100.

27. Rajsekar K, Rajsekar YL, Chaturvedi SK. Psycho ophthalmology: the interface between psychiatry and ophthalmology. Indian J Psychiatry. 1999;41:186-96.

28. Souza ACd, Alexandre NMC, Guirardello EdB. Psychometric properties in instruments evaluation of reliability and validity. Epidemiol Serv Saude. 2017:26:649-59.

\section{Publisher's Note}

Springer Nature remains neutral with regard to jurisdictional claims in published maps and institutional affiliations.

Ready to submit your research? Choose BMC and benefit from

- fast, convenient online submission

- thorough peer review by experienced researchers in your field

- rapid publication on acceptance

- support for research data, including large and complex data types

- gold Open Access which fosters wider collaboration and increased citations

- maximum visibility for your research: over $100 \mathrm{M}$ website views per year

At BMC, research is always in progress.

Learn more biomedcentral.com/submissions 